\title{
On the products of Hecke $L$-functions of holomorphic cusp forms
}

\author{
by \\ Bogdan SzydŁo (Poznań)
}

1. Introduction. There are many reasons to study the values of specific $L$-functions at special points of the complex plane; cf. [1], [4], [7], [8], [14]. For example, for various applications it is necessary to know that there are infinitely many members of a relevant class of $L$-functions which do not vanish at a special point.

In the present paper we consider the aggregates

$$
\mathcal{A}_{N}(u, v ; k)=\sum_{j=1}^{\vartheta(k)} \alpha_{j, k} t_{j, k}(N) H_{j, k}(u) H_{j, k}(v)
$$

of products of the Hecke $L$-functions $H_{j, k}$. Here $u, v \in \mathbb{C}, N \in \mathbb{N}$ and the weights $2 k$ of holomorphic cusp forms vary over even integers $\geq 12$; for notation used, see Section 3.

Some relevance of the binary additive divisor problems to our subject is to be noticed. A spectral approach to these classical problems is given in the fundamental paper [12] by Y. Motohashi. This paper and the monograph [13] by the same author are our main references.

A brief description of Motohashi's method in the context of the dual binary additive divisor problem is given in Section 4.1, where a spectral decomposition for a weighted sum

$$
B_{N}\left(\alpha, \beta ; W_{0}\right)=\sum_{n=1}^{N-1} \sigma_{\alpha}(n) \sigma_{\beta}(N-n) W_{0}(n / N)
$$

is described in some detail. Here $N \in \mathbb{N}, \alpha, \beta \in \mathbb{C}$ and $W_{0}:(0,1) \rightarrow \mathbb{C}$ is a weight function. In the present paper we do not discuss the nature of such a decomposition; instead we refer the reader to [12]. It is sufficient for us to notice that all holomorphic cusp forms contribute to a part of the spectral decomposition. Following [12] this part is denoted by $B_{\mathrm{h}}$ and called

2000 Mathematics Subject Classification: 11F66, 11F67, 11F72, 11M41, $11 \mathrm{~N} 75$. 
holomorphic. From the formula (57) below, it is clear that the $B_{\mathrm{h}}$ is built from the aggregates $\mathcal{A}_{N}$.

If we study $B_{N}\left(\alpha, \beta ; W_{0}\right)$ with $\alpha=\beta$, then the choice

$$
W_{0}(x)=W_{p}(x)= \begin{cases}(x(1-x))^{p-1} & \text { if } 0<x<1 \\ 0 & \text { if } x \geq 1\end{cases}
$$

where $p \in \mathbb{C}$, seems to be natural enough. An explicit form of the holomorphic part $B_{\mathrm{h}}$ of the spectral decomposition for $B_{N}\left(-\varrho,-\varrho ; W_{p}\right)$ (with $0<\Re \varrho<1 / 2$ and $\Re p$ large enough) is given by the series $\mathfrak{H}_{N}(p, \varrho)$ defined in (1) below; cf. (96). In the present paper we solve the problem of analytic continuation of $\mathfrak{H}_{N}(p, \varrho)$ to the left of the complex $p$-plane; cf. also Remark 1 in Section 2.

We describe briefly our main results, the Theorem and Corollaries 1 and 2 from Section 2. We prove that $\mathfrak{H}_{N}(p, \varrho)$ has an arithmetical representation; see the Theorem. From it we obtain a meromorphic continuation of $\mathfrak{H}_{N}(p, \varrho)$ to the whole complex $p$-plane; see Corollary 1. A non-vanishing result for $\mathcal{A}_{N}(1 / 2,1 / 2+\varrho ; k)$ is derived; see Corollary 2 .

The organization of the paper is as follows. In Section 2 the precise statements of the main results are given. We prove them in Section 4. In the intermediate Section 3 some general notation and facts from hypergeometric function theory are collected. In the rest of the present introductory section our method of proof is outlined.

Motohashi's method is sketched in Section 4.1. We use it in Section 4.2 to obtain a spectral decomposition for $B_{N}\left(\alpha, \beta ; W_{0}\right)$, provided that $(\alpha, \beta)$ belongs to a sufficiently small neighbourhood of $(-\varrho,-\varrho)(0<\Re \varrho<1 / 2)$ and $W_{0}$ is nice enough (see the beginning of Section 4.2). If $\alpha=\beta=-\varrho$ and $W_{0}$ is even (i.e. $W_{0}$ satisfies the condition (51) below), the decomposition obtained, except for the holomorphic part $B_{\mathrm{h}}$, resolves itself into two equal, arithmetically significant parts. Using these observations, we separate $B_{\mathrm{h}}$ from the spectral decomposition. For a precise statement, see Proposition 1.

In Section 4.3, by reversing the order of steps in Motohashi's method in the dual additive divisor problem, we express $B_{\mathrm{h}}$ in arithmetical terms, provided that $\alpha=\beta=-\varrho, W_{0}$ is nice enough, even and satisfies additionally the condition (58) (see Propositions 2 and 3 ).

Because of the requirement (58), the findings of Section 4.3 cannot be directly applied to $W_{p}$. In Section 4.4 we study instead finite linear combinations of $W_{p}$-functions. We arrive at Proposition 4 which is a weaker version of our Theorem.

In Section 4.5 we show that the central equality (7) of the Theorem holds for two special values of the argument $p$; see Lemma 5. Such concrete verifications are possible because we have arithmetical expressions for the aggregates $\mathcal{A}_{N}$; see [2], [3], [7], [10], [11]. Relevant formulas are con- 
sequences of classical Petersson's formulas, for which one may consult [13, Lemma 2.3].

From Proposition 4 and Lemma 5 we get our Theorem directly. The discussion in Section 4.5 also yields Corollaries 1 and 2.

We recall that $\mathfrak{H}_{N}(p, \varrho)$, defined by $(1)$, is identical with the holomorphic part $B_{\mathrm{h}}$ of the spectral decomposition for the binary additive divisor sum $B_{N}\left(\alpha, \beta ; W_{0}\right)$ with $\alpha=\beta=-\varrho$ and $W_{0}=W_{p}$. The form of this decomposition is almost symmetrical, but not symmetrical, and therefore enables us to separate $B_{\mathrm{h}}$ from it. This phenomenon of spectral asymmetry seems to be of central importance to the proof. It can be traced back to the use of Kuznetsov's sum formulas in Motohashi's paper [12]. For a thorough presentation of this important tool of modern analytic number theory, one may consult [6] and [13].

Acknowledgments. I thank the referees for valuable remarks on the earlier versions of the paper and Mr Jerzy Trzeciak for a great number of suggestions for the improvement of the presentation of my work.

2. Statement of the results. Let $N \in \mathbb{N}, p, \varrho \in \mathbb{C}$. Under appropriate assumptions (see the Theorem below) and notations (see Section 3 ), put

$$
\begin{aligned}
\mathfrak{H}_{N}(p, \varrho)= & \pi^{-\varrho} 2^{-2 p+\varrho} N^{1 / 2-\varrho} \\
& \times \sum_{k=6, k \text { even }}^{\infty} \frac{\Gamma(p) \Gamma(p-\varrho) \Gamma((k+\varrho) / 2)}{\Gamma(p+(k-\varrho) / 2) \Gamma((1+k-\varrho) / 2)} \\
& \times \Gamma(-p+(1+k+\varrho) / 2) \sin (\pi(p+(1-\varrho) / 2)) \\
& \times \sum_{j=1}^{\vartheta(k)} \alpha_{j, k} t_{j, k}(N) H_{j, k}(1 / 2) H_{j, k}(1 / 2+\varrho), \\
\mathfrak{G}_{N}(p, \varrho)= & \frac{\cot (\pi \varrho / 2)}{\pi}\left(N^{-\varrho} \sigma_{-\varrho}(N) \zeta(-\varrho) \frac{\Gamma(p) \Gamma(p-\varrho-1)}{\Gamma(2 p-\varrho-1)}\right. \\
& \left.-\sigma_{-\varrho}(N) \zeta(\varrho) \frac{\Gamma(p-1) \Gamma(p-\varrho)}{\Gamma(2 p-\varrho-1)}\right)
\end{aligned}
$$

where

$$
\begin{aligned}
A_{p, \varrho}(x)= & \frac{\cot (\pi \varrho / 2)}{\pi} \frac{\Gamma(p) \Gamma(p-\varrho)}{\Gamma(2 p-\varrho)}\left(F(1, p-\varrho ; 2 p-\varrho ;-1 / x) x^{\varrho-1}\right. \\
& \left.-F(1, p ; 2 p-\varrho ;-1 / x)(1+x)^{\varrho} x^{-1}\right) \quad(x>0),
\end{aligned}
$$




$$
\begin{aligned}
D_{p, \varrho}(x)= & (1+\cot (\pi \varrho / 2) \cot (\pi p))(x(1-x))^{p-1} \\
& -\frac{\cot (\pi \varrho / 2)}{\pi} \frac{\Gamma(p-\varrho) \Gamma(p-1)}{\Gamma(2 p-\varrho-1)} \\
& \times(1-x)^{\varrho} F(1,2+\varrho-2 p ; 2-p ; x) \quad(0<x<1) .
\end{aligned}
$$

Theorem. Let $N \in \mathbb{N}, \varrho, p \in \mathbb{C}$. Assume that

$$
0<\Re \varrho<1 / 2, \quad \Re p>3 / 2+\Re \varrho / 2 .
$$

Then

$$
\mathfrak{H}_{N}(p, \varrho)=\mathfrak{G}_{N}(p, \varrho)+\mathfrak{A}_{N}(p, \varrho)+\mathfrak{D}_{N}(p, \varrho) .
$$

Fix $\varrho \in \mathbb{C}$ with $0<\Re \varrho<1 / 2$. Consider both sides of (7) as functions of the complex parameter $p$. From the Theorem and the discussion in Section 4.5 we obtain

COROLlaRY 1. The function $\mathfrak{H}_{N}(p, \varrho)$ continues meromorphically from the halfplane $\Re p>3 / 2+\Re \varrho / 2$ to the whole complex plane $\mathbb{C}$. The continuation is given by $\mathfrak{G}_{N}(p, \varrho)+\mathfrak{A}_{N}(p, \varrho)+\mathfrak{D}_{N}(p, \varrho)$. It has only two poles in the halfplane $\Re p>1 / 2$, at $p=1+\varrho$ and $p=1$. They are simple and

$$
\begin{gathered}
\underset{p=1+\varrho}{\operatorname{Res}} \mathfrak{H}_{N}(p, \varrho)=\frac{\cot (\pi \varrho / 2)}{\pi} N^{-\varrho} \sigma_{-\varrho}(N) \zeta(-\varrho), \\
\operatorname{Res}_{p=1} \mathfrak{H}_{N}(p, \varrho)=-\frac{\cot (\pi \varrho / 2)}{\pi} \sigma_{-\varrho}(N) \zeta(\varrho) .
\end{gathered}
$$

Corollary 2. Let $N \in \mathbb{N}$ and $\varrho \in \mathbb{C}$ with $0<\Re \varrho<1 / 2$. Then there are infinitely many even integers $k$ such that

$$
\sum_{j=1}^{\vartheta(k)} \alpha_{j, k} t_{j, k}(N) H_{j, k}(1 / 2) H_{j, k}(1 / 2+\varrho) \neq 0 .
$$

Proof. Suppose the contrary. Then the sum in (1) is finite and the function $\mathfrak{H}_{N}(p, \varrho) /(\Gamma(p) \Gamma(p-\varrho))$ is regular on $\mathbb{C}$. Since $\operatorname{Res}_{p=1+\varrho} \mathfrak{H}_{N}(p, \varrho) \neq 0$, this is impossible.

Remarks. 1. As stated in the introduction, $\mathfrak{H}_{N}(p, \varrho)$ is the holomorphic part $B_{\mathrm{h}}$ of the spectral decomposition for the binary additive divisor sum $B_{N}\left(-\varrho,-\varrho ; W_{p}\right)$ (see (1) and (96)) and, as such, it can be represented by $B_{N}\left(-\varrho,-\varrho ; W_{p}\right)$ and the other parts of the decomposition; see (29)-(31), (38), (39) below and, for further details, [12]. However, because of the convergence problems, such a spectral representation gives no analytic continuation of $\mathfrak{H}_{N}(p, \varrho)$ to the left of the complex $p$-plane. 
2. Put $p=1 / 2+\varrho / 2+l, l$ being an integer $\geq 2$. Then $\mathfrak{H}_{N}(p, \varrho)$ simplifies to a finite linear combination of the aggregates

$$
\mathcal{A}_{N}(1 / 2,1 / 2+\varrho ; k)=\sum_{j=1}^{\vartheta(k)} \alpha_{j, k} t_{j, k}(N) H_{j, k}(1 / 2) H_{j, k}(1 / 2+\varrho) .
$$

It was mentioned in the introduction that there are arithmetical expressions for $\mathcal{A}_{N}$; see [2], [3], [7], [10], [11]. In fact, we deduce the Theorem from its weaker version, Proposition 4, by using the above observations; cf. Section 4.5.

3. Let us note that the aggregates

$$
\mathcal{A}_{N}(1 / 2,1 / 2 ; k)=\sum_{j=1}^{\vartheta(k)} \alpha_{j, k} t_{j, k}(N) H_{j, k}^{2}(1 / 2)
$$

are also an object of some recent research; see, for example, [7]. These aggregates form the function $\mathfrak{H}_{N}(p, 0)$ (in (1) let $\varrho=0$ ).

It is evident that from the results stated above and concerning $\mathfrak{H}_{N}(p, \varrho)$, $0<\Re \varrho<1 / 2$, we can derive those for $\mathfrak{H}_{N}(p, 0)$ by letting $\varrho \rightarrow 0$.

3. Notation. Facts from hypergeometric function theory. We use the standard notations

$$
\begin{gathered}
e(x)=e^{2 \pi i x}, \quad \sigma_{s}(n)=\sum_{d \mid n} d^{s}, \quad \int_{(a)} \ldots d s=\int_{a-i \infty}^{a+i \infty} \ldots d s, \\
S(m, n ; l)=\sum_{1 \leq h \leq l,(h, l)=1} e\left(\left(m h+n h^{*}\right) / l\right), \quad h h^{*} \equiv 1(\bmod l) .
\end{gathered}
$$

From the theory of modular forms we use just a few conventions; for more information see, for example, [13]. Let $\left(\varphi_{j, k}\right)_{1 \leq j \leq \vartheta(k)}$ denote the orthonormal base of the Petersson unitary space of holomorphic cusp forms of weight $2 k$ with respect to $\mathrm{SL}_{2}(\mathbb{Z})$. It is assumed that $\varphi_{j, k}$ are eigenfunctions of all Hecke operators $T_{k}(n)$ with eigenvalues $t_{j, k}(n), n \geq 1$. Let $\varrho_{j, k}(1)$ be the first Fourier coefficient of $\varphi_{j, k}$. Set

$$
\alpha_{j, k}=16 \Gamma(2 k-1)(4 \pi)^{-2 k-1}\left|\varrho_{j, k}(1)\right|^{2} .
$$

The Hecke $L$-function attached to $\varphi_{j, k}$ is defined by

$$
H_{j, k}(s)=\sum_{n=1}^{\infty} t_{j, k}(n) n^{-s} \quad(\Re s>1) .
$$

We need the following facts from the theory of the Gauss hypergeometric function $F(a, b ; c ; z)(a, b, c, z \in \mathbb{C} ; c \neq 0,-1,-2, \ldots)$ : 
(8) $\quad F(a, b ; c ; z)=\sum_{n=1}^{\infty} \frac{\Gamma(c) \Gamma(a+n) \Gamma(b+n)}{\Gamma(a) \Gamma(b) \Gamma(c+n) \Gamma(n+1)} z^{n} \quad(|z|<1)$,

$$
F(a, b ; c ; z)=\frac{\Gamma(c)}{\Gamma(b) \Gamma(c-b)} \int_{0}^{1} t^{b-1}(1-t)^{c-b-1}(1-t z)^{-a} d t
$$

$(\Re c>\Re b>0 ;|\arg (1-z)|<\pi)$,

(10) $\quad F(a, b ; c ; z)=\frac{\Gamma(c)}{\Gamma(a) \Gamma(b)} \frac{1}{2 \pi i} \int_{(\gamma)} \frac{\Gamma(s) \Gamma(a-s) \Gamma(b-s)}{\Gamma(c-s)}(-z)^{-s} d s$

(11) $\quad F(a, b ; c ; z)=\frac{\Gamma(c)}{\Gamma(a) \Gamma(b) \Gamma(c-a) \Gamma(c-b)}$

$$
(0<\gamma<\Re a, \Re b ;|\arg (-z)|<\pi),
$$

$$
\begin{array}{r}
\times \frac{1}{2 \pi i} \int_{(\gamma)} \Gamma(s) \Gamma(s+c-a-b) \Gamma(a-s) \Gamma(b-s)(1-z)^{-s} d s \\
(0, \Re(a+b-c)<\gamma<\Re a, \Re b ;|\arg (1-z)|<\pi),
\end{array}
$$

$$
\begin{aligned}
F(a, b ; c ; z)= & \frac{\Gamma(c) \Gamma(c-a-b)}{\Gamma(c-a) \Gamma(c-b)} F(a, b ; 1+a+b-c ; 1-z) \\
& +\frac{\Gamma(c) \Gamma(a+b-c)}{\Gamma(a) \Gamma(b)} \\
& \times(1-z)^{c-a-b} F(c-a, c-b ; 1+c-a-b ; 1-z) \\
& \quad(a+b-c \notin \mathbb{Z} ;|\arg (1-z)|<\pi),
\end{aligned}
$$

(13) $\quad F(a, b ; c ; z)=(1-z)^{-a} F(a, c-b ; c ; z /(z-1))$

$$
=(1-z)^{c-a-b} F(c-a, c-b ; c ; z) \quad(|\arg (1-z)|<\pi)
$$

(see, for example, [5, Chapter 2]). It is easy to see that $(a, b, c, z \in \mathbb{C} ; b \neq 1$; $c \neq 0,-1,-2, \ldots ;|\arg (1-z)|<\pi)$

$$
\begin{aligned}
& F(a, 0 ; c ; z)=1 \\
& F(a, c ; c ; z)=(1-z)^{-a} \\
& F(1, b ; 2 ; z)=\frac{1}{(b-1) z}\left((1-z)^{1-b}-1\right) .
\end{aligned}
$$

We need the quadratic transformation

$$
\begin{aligned}
& F(2 a, 2 b ; a+b+1 / 2 ;(1-z) / 2) \\
& =\frac{\Gamma(a+b+1 / 2) \Gamma(1 / 2)}{\Gamma(a+1 / 2) \Gamma(b+1 / 2)} F\left(a, b ; 1 / 2 ; z^{2}\right) \\
& +\frac{\Gamma(a+b+1 / 2) \Gamma(-1 / 2)}{\Gamma(a) \Gamma(b)} z F\left(a+1 / 2, b+1 / 2 ; 3 / 2 ; z^{2}\right) \\
& (a+b+1 / 2 \neq 0,-1,-2, \ldots ;|\arg (1 \pm z)|<\pi)
\end{aligned}
$$

(see $[5,2.11(3)])$. 
The integral

$$
\begin{array}{r}
\int_{0}^{1} x^{\alpha-1}(1-x)^{\beta-1} F(a, b ; c ; x) d x=\frac{\Gamma(\alpha) \Gamma(\beta)}{\Gamma(\alpha+\beta)}{ }_{3} F_{2}(a, b, \alpha ; c, \alpha+\beta ; 1) \\
(\Re \alpha, \Re \beta, \Re(c-a-b+\beta)>0)
\end{array}
$$

is easy to check. Here the value at $z=1$ of ${ }_{3} F_{2}\left(a_{1}, a_{2}, a_{3} ; b_{1}, b_{2} ; z\right)$ is used. For ${ }_{p} F_{q}$-functions, see [5, Chapter 4].

We also need

$$
\begin{aligned}
& { }_{3} F_{2}(a, b, c ;(a+b+1) / 2,2 c ; 1) \\
& =\frac{\Gamma(1 / 2) \Gamma(c+1 / 2) \Gamma((a+b+1) / 2) \Gamma((1-a-b) / 2+c)}{\Gamma((a+1) / 2) \Gamma((b+1) / 2) \Gamma((1-a) / 2+c) \Gamma((1-b) / 2+c)} \\
& \quad(\Re(2 c-a-b)>-1)
\end{aligned}
$$

(see $[5,4.4(6)])$.

\section{Proof of Theorem}

4.1. Motohashi's method in the dual additive divisor problem. In the context of the dual additive divisor problems Motohashi [12] investigates the following sums:

$$
B_{N}\left(\alpha, \beta ; W_{0}\right)=\sum_{n=1}^{N-1} \sigma_{\alpha}(n) \sigma_{\beta}(N-n) W_{0}(n / N) .
$$

Here $N \in \mathbb{N}, \alpha, \beta \in \mathbb{C}$ and the weight function $W_{0}:(0,1) \rightarrow \mathbb{C}$ is a smooth function with compact support. Motohashi shows a spectral decomposition of $B_{N}\left(\alpha, \beta ; W_{0}\right)$ in the domain

$$
R(b)=\left\{(\alpha, \beta) \in \mathbb{C}^{2}: 0>\Re \alpha>b, 2 b-2>\Re(\alpha+\beta)\right\},
$$

where $b$ is an arbitrary fixed negative number (see [12, Section 3]). In Section 4 of [12] the decomposition obtained is continued analytically to a neighbourhood of $(0,0) \in \mathbb{C}^{2}$.

We will briefly sketch Motohashi's method (see also N. V. Kuznetsov's earlier publication [10]). Our notation is close to [12]. Moreover, sometimes we will cite some formulas without referring to [12] explicitly.

For $\Re \nu<0$ and $n \in \mathbb{N}$ we have an identity of Ramanujan

$$
\sigma_{\nu}(n)=\zeta(1-\nu) \sum_{l=1}^{\infty} l^{\nu-1} c_{l}(n)
$$

where

$$
c_{l}(n)=\sum_{1 \leq h \leq l,(h, l)=1} e(h n / l)
$$


The Mellin transform of $W_{0}$ is denoted by

$$
w_{0}(s)=\int_{0}^{1} W_{0}(x) x^{s-1} d x \quad(s \in \mathbb{C}),
$$

and its inversion is

$$
W_{0}(x)=\frac{1}{2 \pi i} \int_{(a)} w_{0}(s) x^{-s} d s \quad(x>0)
$$

with a certain $a \in \mathbb{R}$.

Assume that $(\alpha, \beta) \in R(b)$. The application of (22), (23) and (25) transforms (20) into

$$
\begin{aligned}
B_{N}\left(\alpha, \beta ; W_{0}\right)= & \zeta(1-\beta) \sum_{l=1}^{\infty} l^{\beta-1} \sum_{1 \leq h \leq l,(h, l)=1} e(-N h / l) \\
& \times \frac{1}{2 \pi i} \int_{(a)} N^{s} w_{0}(s) D(s, \alpha ; e(h / l)) d s,
\end{aligned}
$$

where $a>1$ is sufficiently large, and

$$
\begin{aligned}
& D(s, \alpha ; e(h / l))=\sum_{n=1}^{\infty} \sigma_{\alpha}(n) e(h n / l) n^{-s} \\
& \quad((h, l)=1, \Re s>1+\max (0, \Re \alpha))
\end{aligned}
$$

is the Estermann-Hecke zeta function (the $D$-function).

The principal analytical properties of the $D$-function are given in the following lemma; see, for example, [13, Lemma 3.7].

Lemma 1. For each fixed $\alpha \neq 0$ the function $D(s, \alpha ; e(h / l))$ is a meromorphic function of $s$, which has simple poles at $s=1$ and $s=1+\alpha$ with residues $\zeta(1-\alpha) l^{\alpha-1}$ and $\zeta(1+\alpha) l^{-\alpha-1}$, respectively; it is regular elsewhere. It satisfies the functional equation

$$
\begin{aligned}
D(s, \alpha ; e(h / l))= & 2(2 \pi)^{2 s-2-\alpha} l^{\alpha-2 s+1} \Gamma(1-s) \Gamma(1+\alpha-s) \\
& \times\left\{\cos (\pi \alpha / 2) D\left(1-s,-\alpha ; e\left(h^{*} / l\right)\right)\right. \\
& \left.-\cos (\pi(s-\alpha / 2)) D\left(1-s,-\alpha ; e\left(-h^{*} / l\right)\right)\right\},
\end{aligned}
$$

where $h h^{*} \equiv 1(\bmod l)$. The function $D(s, \alpha ; e(h / l))$ is of polynomial order with respect to $s$ if $\Re$ s is bounded.

Now the integration line in (26) can be translated to $\Re s=b$. The residue theorem gives

$$
B_{N}\left(\alpha, \beta ; W_{0}\right)=V_{1}(\alpha, \beta)+B_{1}(\alpha, \beta) .
$$

Here $V_{1}(\alpha, \beta)$ is the contribution of the poles of the integrands in (26) at $s=1$ and $s=1+\alpha$ (see Lemma 1 ) and $B_{1}(\alpha, \beta)$ is given by the right hand side of (26) but with the new integration line $\Re s=b$. 
Lemma 1, (22) and (23) imply that

$$
\begin{aligned}
V_{1}(\alpha, \beta)= & N^{\alpha+\beta} \sigma_{1-\alpha-\beta}(N) \frac{\zeta(1-\alpha) \zeta(1-\beta)}{\zeta(2-\alpha-\beta)} w_{0}(1) \\
& +N^{\beta} \sigma_{1+\alpha-\beta}(N) \frac{\zeta(1+\alpha) \zeta(1-\beta)}{\zeta(2+\alpha-\beta)} w_{0}(1+\alpha) .
\end{aligned}
$$

The functional equation (28) of the $D$-function and its definition (27) give

$$
B_{1}(\alpha, \beta)=B_{1}^{+}(\alpha, \beta)+B_{1}^{-}(\alpha, \beta),
$$

where

$$
\begin{aligned}
B_{1}^{+}(\alpha, \beta) & \\
= & -\zeta(1-\beta) \sum_{l=1}^{\infty} l^{\beta-1} \sum_{1 \leq h \leq l,(h, l)=1} e(-N h / l) \\
\times & \frac{1}{2 \pi i} \int_{(b)} N^{s} w_{0}(s) 2(2 \pi)^{2 s-2-\alpha} l^{\alpha-2 s+1} \Gamma(1-s) \Gamma(1+\alpha-s) \\
\times & \cos (\pi(s-\alpha / 2)) D\left(1-s,-\alpha ; e\left(-h^{*} / l\right)\right) d s \\
B_{1}^{-}(\alpha, \beta)= & \zeta(1-\beta) \sum_{l=1}^{\infty} l^{\beta-1} \sum_{1 \leq h \leq l,(h, l)=1} e(-N h / l) \\
& \times \frac{\cos (\pi \alpha / 2)}{2 \pi i} \int_{(b)} N^{s} w_{0}(s) 2(2 \pi)^{2 s-2-\alpha} l^{\alpha-2 s+1} \\
& \times \Gamma(1-s) \Gamma(1+\alpha-s) D\left(1-s,-\alpha ; e\left(h^{*} / l\right)\right) d s
\end{aligned}
$$

and further

$$
\begin{aligned}
B_{1}^{ \pm}(\alpha, \beta)= & 2(2 \pi)^{\beta-1} \zeta(1-\beta) N^{(\alpha+\beta+1) / 2} \\
& \times \sum_{n=1}^{\infty} \sigma_{-\alpha}(n) n^{(\alpha+\beta-1) / 2} L_{ \pm}(N, n ; \alpha, \beta),
\end{aligned}
$$

where

$$
L_{ \pm}(N, n ; \alpha, \beta)=\sum_{l=1}^{\infty} \frac{1}{l} S( \pm N, n ; l) \varphi_{ \pm}(4 \pi \sqrt{N n} / l ; \alpha, \beta)
$$

with $(x>0)$

$$
\begin{aligned}
\varphi_{+}(x ; \alpha, \beta)= & -\frac{1}{2 \pi i} \int_{(b)} \Gamma(1-s) \Gamma(1+\alpha-s) \cos (\pi(s-\alpha / 2)) \\
& \times w_{0}(s)(x / 2)^{2 s-\alpha-\beta-1} d s \\
\varphi_{-}(x ; \alpha, \beta)= & \frac{\cos (\pi \alpha / 2)}{2 \pi i} \int_{(b)} \Gamma(1-s) \Gamma(1+\alpha-s) \\
& \times w_{0}(s)(x / 2)^{2 s-\alpha-\beta-1} d s .
\end{aligned}
$$


The formulas (29)-(37) show that the sum $B_{N}\left(\alpha, \beta ; W_{0}\right)$ reduces to a double sum of the Kloosterman sums $S( \pm N, n ; l)$. Kuznetsov's sum formulas (see [9], [12, Lemmas 1 and 2] or [13, Chapter 2]) are now applied to the inner sums $L_{ \pm}(N, n ; \alpha, \beta)$. The assumption $(\alpha, \beta) \in R(b)$ is needed just at this stage of Motohashi's procedure. The resulting spectral expressions can be summed with the help of another formula by Ramanujan $(\mu, \nu \in \mathbb{C} ; \Re s$ large enough)

$$
\sum_{n=1}^{\infty} \sigma_{\mu}(n) \sigma_{\nu}(n) n^{-s}=\zeta(s) \zeta(s-\mu) \zeta(s-\nu) \zeta(s-\mu-\nu) / \zeta(2 s-\mu-\nu)
$$

and its appropriate analogs; cf. [12, (2.3), (2.4), (2.9)].

In this way a spectral decomposition of $B_{N}\left(\alpha, \beta ; W_{0}\right)$ is obtained, provided that $(\alpha, \beta) \in R(b)$; see [12, Section 3]. In Section 4 of [12] Motohashi shows how to continue analytically this decomposition to a neighbourhood of $(0,0) \in \mathbb{C}^{2}$.

In particular, if $\alpha$ and $\beta$ are sufficiently small and $\alpha \pm \beta \neq 0$ one has

$$
\begin{aligned}
B_{1}^{+}(\alpha, \beta)= & V_{2}^{+}(\alpha, \beta)+V_{3}^{+}(\alpha, \beta)+\widetilde{B}_{\mathrm{c}}^{+}(\alpha, \beta)+B_{\mathrm{d}}^{+}(\alpha, \beta) \\
& +B_{\mathrm{h}}(\alpha, \beta), \\
B_{1}^{-}(\alpha, \beta)= & V_{2}^{-}(\alpha, \beta)+V_{3}^{-}(\alpha, \beta)+\widetilde{B}_{\mathrm{c}}^{-}(\alpha, \beta)+B_{\mathrm{d}}^{-}(\alpha, \beta)
\end{aligned}
$$

(see (29)-(31)). Explicit forms of the terms $V_{2}^{ \pm}(\alpha, \beta), V_{3}^{ \pm}(\alpha, \beta), \widetilde{B}_{\mathrm{c}}^{ \pm}(\alpha, \beta)$, and $B_{\mathrm{h}}(\alpha, \beta)$ can be found in $[12,(3.58),(4.33),(4.34)]$. For $B_{\mathrm{d}}^{ \pm}(\alpha, \beta)$, see $\left[12,(3.58)_{\mathrm{d}}\right]$, where

$$
B_{\mathrm{d}}(\alpha, \beta)=B_{\mathrm{d}}^{+}(\alpha, \beta)+B_{\mathrm{d}}^{-}(\alpha, \beta)
$$

is displayed. The lower indices $\mathrm{c}, \mathrm{d}$ and $\mathrm{h}$ in the above formulas are used to signal the fact that a given term depends on the continuous, discrete or holomorphic part of the spectrum of the hyperbolic Laplacian, respectively.

4.2. Spectral asymmetry. In order to prove the Theorem we use the just sketched Motohashi Ansatz in the additive divisor problem (see Section 4.1).

First of all we replace the assumptions on the weight function $W_{0}$ by weaker ones; cf. Motohashi's remark after Theorem 4 in [12].

We say that a function $W_{0}:(0,1) \rightarrow \mathbb{C}$ is nice enough if $W_{0}$ is smooth,

$$
\lim _{x \rightarrow 0+} W_{0}(x)=0, \quad \lim _{x \rightarrow 1-} W_{0}(x)=0,
$$

and the order of decay of $W_{0}$ at $x=0$ and $x=1$ is sufficiently high.

Further on we assume that the function $W_{0}$ is nice enough. We also fix $\varrho \in \mathbb{C}$ with $0<\Re \varrho<1 / 2$. 
By modifying the argument in Sections 3 and 4 of [12] one can show that $B_{N}\left(\alpha, \beta ; W_{0}\right)$ given by (20) and (29)-(37) can be spectrally decomposed in the domain $R(-1 / 2)$ (see $(21)$ ), and then analytically continued to a neighbourhood of $(-\varrho,-\varrho) \in \mathbb{C}^{2}$. In particular, provided that $(\alpha, \beta)$ belongs to a sufficiently small neighbourhood of $(-\varrho,-\varrho)$, we have there the spectral decomposition for $B_{1}^{ \pm}(\alpha, \beta)$ of the type (38) and (39), the terms $\widetilde{B}_{\mathrm{c}}^{ \pm}(\alpha, \beta)$, $B_{\mathrm{d}}^{ \pm}(\alpha, \beta)$ and $B_{\mathrm{h}}(\alpha, \beta)$ being regular. For the terms $V_{2}^{ \pm}(\alpha, \beta)+V_{3}^{ \pm}(\alpha, \beta)$, we have the expressions (4.33) and (4.34) in [12]; cf. also (44) below. But these expressions are valid only under the additional assumption

$$
\alpha \neq \beta \text {. }
$$

This assumption can be dispensed with. To show this, more details from [12] are needed.

In $[12,(3.46),(3.52)$ and (3.48)] the following three functions of complex variables $\xi, u$ and $v$ are introduced:

$$
\begin{aligned}
& \Phi_{+}(\xi ; u, v)= \frac{-1}{4 \pi i} \int_{-i \infty}^{+i \infty} \cos (\pi(s-u / 2)) \sin (\pi(s-(u+v) / 2)) \\
& \times \Gamma(s-(u+v+1) / 2+\xi) \Gamma(s-(u+v+1) / 2-\xi) \\
& \times \Gamma(1-s) \Gamma(1+u-s) w_{0}(s) d s \\
& \Phi_{-}(\xi ; u, v) \\
&=\frac{1}{4 \pi i} \cos (\pi \xi) \cos (\pi u / 2) \int_{-i \infty}^{+i \infty} \Gamma(s-(u+v+1) / 2+\xi) \\
& \times \Gamma(s-(u+v+1) / 2-\xi) \Gamma(1-s) \Gamma(1+u-s) w_{0}(s) d s \\
& \Xi_{+}(\xi ; u, v)= \frac{1}{2 \pi i} \int_{-i \infty}^{+i \infty} \frac{\Gamma(\xi-(u+v+1) / 2+s)}{\Gamma(\xi+(u+v+3) / 2-s)} \\
& \times \Gamma(1-s) \Gamma(1+u-s) w_{0}(s) \cos (\pi(s-u / 2)) d s .
\end{aligned}
$$

In (41) and (42) it is assumed that

$$
(1-v \pm u) / 2 \pm \xi \neq 0,-1,-2, \ldots
$$

The path of integration starts from $-i \infty$ and ends at $+i \infty$ in such a way that the poles of $\Gamma(s-(u+v+1) / 2 \pm \xi)$ lie to the left and those of $\Gamma(1-s) \Gamma(1+$ $u-s)$ to the right of it. Under the above assumptions on the variables $\xi, u$ and $v$, such a path exists. In (43) it is assumed that

$$
(1-v \pm u) / 2+\xi \neq 0,-1,-2, \ldots
$$

Here the path of integration separates the poles of $\Gamma(s-(u+v+1) / 2+\xi)$ from the poles of $\Gamma(1-s) \Gamma(1+u-s)$. Such a path exists. We remark that the function $\Phi_{+}$is expressible by means of the function $\Xi_{+}$(see $[12,(3.47)]$ ). 
All the terms on the right hand side of (38) and (39) are expressible by means of the function $\Phi_{+}, \Phi_{-}$, respectively, in a symmetric way; see again [12, (3.58), (4.33) and (4.34)]. In particular, for $\alpha$ and $\beta$ sufficiently close to $-\varrho$ and provided that (40) holds, we have

$$
\begin{aligned}
V_{2}^{ \pm}(\alpha, \beta)+ & V_{3}^{ \pm}(\alpha, \beta) \\
= & \frac{2}{\pi \cos (\pi \beta / 2)} \sigma_{\alpha+\beta+1}(N) \frac{\zeta(1+\alpha) \zeta(1+\beta)}{\zeta(2+\alpha+\beta)} \\
& \times \Phi_{ \pm}((\alpha+\beta+1) / 2 ; \alpha, \beta)+\frac{2}{\pi \cos (\pi \beta / 2)} N^{\alpha} \sigma_{-\alpha+\beta+1}(N) \\
& \times \frac{\zeta(1-\alpha) \zeta(1+\beta)}{\zeta(2-\alpha+\beta)} \Phi_{ \pm}((-\alpha+\beta+1) / 2 ; \alpha, \beta) .
\end{aligned}
$$

Note that here the assumption (40) is really necessary if we adopt the definitions (41) and (42) of the functions $\Phi_{ \pm}$. But we have the following lemma (cf. also [10, Supplement to Theorem 3.5]).

Lemma 2. If $W_{0}$ is nice enough, and $\xi, u, v \in \mathbb{C}$ are in generic position, then

$$
\begin{aligned}
& \Phi_{+}(\xi ; u, v) \\
& =\frac{\pi \sin (\pi(u+v) / 2)}{4 \sin (\pi u / 2)} \frac{\Gamma((1-u-v) / 2+\xi) \Gamma((1-u-v) / 2-\xi)}{\Gamma(1-u)} \\
& \times \int_{0}^{1} W_{0}(x) F((1-u-v) / 2+\xi,(1-u-v) / 2-\xi ; 1-u ; x) d x \\
& +\frac{\pi \sin (\pi(u-v) / 2)}{4 \sin (\pi u / 2)} \frac{\Gamma((1+u-v) / 2+\xi) \Gamma((1+u-v) / 2-\xi)}{\Gamma(1+u)} \\
& \times \int_{0}^{1} W_{0}(x) x^{u} F((1+u-v) / 2+\xi,(1+u-v) / 2-\xi ; 1+u ; x) d x, \\
& \text { (46) } \Phi_{-}(\xi ; u, v) \\
& \begin{aligned}
= & \frac{\cos (\pi \xi) \cos (\pi u / 2)}{2} \frac{\Gamma((1-u-v) / 2+\xi) \Gamma((1-u-v) / 2-\xi)}{\Gamma(1-v)} \\
& \times \Gamma((1+u-v) / 2+\xi) \Gamma((1+u-v) / 2-\xi) \\
& \times \int_{0}^{1} W_{0}(x) F((1-u-v) / 2+\xi,(1-u-v) / 2-\xi ; 1-v ; 1-x) d x .
\end{aligned}
\end{aligned}
$$

Proof. Under the assumption that $u \notin \mathbb{Z}$, translate the paths of integration in (41) and (42) to $\Re s=+\infty$. The residue theorem, (24), and (8) give (45) and, in the case of $\Phi_{-}$, 
$\Phi_{-}(\xi ; u, v)$

$$
\begin{aligned}
= & \frac{\pi \cos (\pi \xi)}{4 \sin (\pi u / 2)}\left(\frac{\Gamma((1-u-v) / 2+\xi) \Gamma((1-u-v) / 2-\xi)}{\Gamma(1-u)}\right. \\
& \quad \times \int_{0}^{1} W_{0}(x) F((1-u-v) / 2+\xi,(1-u-v) / 2-\xi ; 1-u ; x) d x \\
& -\frac{\Gamma((1+u-v) / 2+\xi) \Gamma((1+u-v) / 2-\xi)}{\Gamma(1+u)} \\
& \left.\left.\quad \times \int_{0}^{1} W_{0}(x) x^{u} F((1+u-v) / 2+\xi,(1+u-v) / 2-\xi) ; 1+u ; x\right) d x\right) .
\end{aligned}
$$

By (12), this is identical with (46).

Now it is clear from (41)-(46) that the assumption (40) can be dropped and the terms $V_{2}^{ \pm}(\alpha, \beta)+V_{3}^{ \pm}(\alpha, \beta)$ are regular at $(-\varrho,-\varrho)$. This also means that $B_{1}^{ \pm}(\alpha, \beta)$ are regular at $(-\varrho,-\varrho)$.

Moreover, by using (15) and (14), we obtain from (45) and (46)

$$
\begin{aligned}
& \Phi_{+}(1 / 2-\varrho ;-\varrho,-\varrho) \\
&=\frac{\pi \cos (\pi \varrho / 2)}{2} \frac{\Gamma(2 \varrho)}{\Gamma(1+\varrho)} \int_{0}^{1} W_{0}(x) F(1,2 \varrho ; 1+\varrho ; x) d x,
\end{aligned}
$$

$$
\begin{aligned}
\Phi_{+}(1 / 2 ;-\varrho,-\varrho) & \lim _{\alpha \rightarrow-\varrho, \beta \rightarrow-\varrho} \Phi_{+}((\beta-\alpha+1) / 2 ; \alpha, \beta) \\
= & \frac{\pi}{2} \cos \left(\frac{\pi \varrho}{2}\right) \Gamma(\varrho) \int_{0}^{1} W_{0}(x)\left((1-x)^{-\varrho}-\frac{1}{2} x^{-\varrho}\right) d x,
\end{aligned}
$$

$$
\begin{gathered}
\Phi_{-}(1 / 2-\varrho ;-\varrho,-\varrho) \\
=\frac{\pi \cos (\pi \varrho / 2)}{2} \frac{\Gamma(2 \varrho)}{\Gamma(1+\varrho)} \int_{0}^{1} W_{0}(x) F(1,2 \varrho ; 1+\varrho ; 1-x) d x \\
\Phi_{-}(1 / 2 ;-\varrho,-\varrho)=\lim _{\alpha \rightarrow-\varrho, \beta \rightarrow-\varrho} \Phi_{-}((\beta-\alpha+1) / 2 ; \alpha, \beta) \\
=\frac{\pi}{4} \cos \left(\frac{\pi \varrho}{2}\right) \Gamma(\varrho) \int_{0}^{1} W_{0}(x) x^{-\varrho} d x .
\end{gathered}
$$

Now we add a new assumption on $W_{0}$.

We say that $W_{0}:(0,1) \rightarrow \mathbb{C}$ is even (with respect to $\left.1 / 2\right)$ if it satisfies

$$
W_{0}(x)=W_{0}(1-x) \quad(0<x<1) .
$$


If $W_{0}$ is nice enough and even, then from (47)-(51) we have

$$
\begin{aligned}
\Phi_{+}(1 / 2-\varrho ;-\varrho,-\varrho) & =\Phi_{-}(1 / 2-\varrho ;-\varrho,-\varrho), \\
\Phi_{+}(1 / 2 ;-\varrho,-\varrho) & =\Phi_{-}(1 / 2 ;-\varrho,-\varrho) .
\end{aligned}
$$

This and (44) give

$$
V_{2}^{+}(-\varrho,-\varrho)+V_{3}^{+}(-\varrho,-\varrho)=V_{2}^{-}(-\varrho,-\varrho)+V_{3}^{-}(-\varrho,-\varrho) .
$$

Observe also that (45), (46) and (51) imply even more easily that

$$
\Phi_{+}(\xi ;-\varrho,-\varrho)=\Phi_{-}(\xi ;-\varrho,-\varrho) \quad(\Re \xi=0) .
$$

From the explicit expressions for $\widetilde{B}_{\mathrm{c}}^{ \pm}(\alpha, \beta)$ and $B_{\mathrm{d}}^{ \pm}(\alpha, \beta)$ (see [12, (3.58)]), and (53) we get

$$
\widetilde{B}_{\mathrm{c}}^{+}(-\varrho,-\varrho)=\widetilde{B}_{\mathrm{c}}^{-}(-\varrho,-\varrho), \quad B_{\mathrm{d}}^{+}(-\varrho,-\varrho)=B_{\mathrm{d}}^{-}(-\varrho,-\varrho) .
$$

Finally, recall the representations (38) and (39) for $B_{1}^{ \pm}(\alpha, \beta)$, and use (52) and (54) to obtain

Proposition 1. Define

$$
B^{\bullet}(\alpha, \beta)=B_{1}^{+}(\alpha, \beta)-B_{1}^{-}(\alpha, \beta) \quad((\alpha, \beta) \in R(-1 / 2)),
$$

where $B_{1}^{ \pm}(\alpha, \beta)$ are given by (32) and (33). If the weight function $W_{0}$ is nice enough, then $B^{\bullet}$ has an analytic continuation from the domain $R(-1 / 2)$ to a neighbourhood of $(-\varrho,-\varrho) \in \mathbb{C}^{2}$. If $W_{0}$ is also even (see (51)), then

$$
B^{\bullet}(-\varrho,-\varrho)=B_{\mathrm{h}}(-\varrho,-\varrho) .
$$

From $\left[12,(3.58)_{\mathrm{h}}\right]$ we quote (correcting a misprint) the explicit formula for $B_{\mathrm{h}}(\alpha, \beta)$ :

$$
\begin{aligned}
& B_{\mathrm{h}}(\alpha, \beta) \\
& =\frac{1}{2}(2 \pi)^{\beta} N^{(\alpha+\beta+1) / 2} \sum_{k=6}^{\infty} \sum_{j=1}^{\vartheta(k)}(-1)^{k-1} \alpha_{j, k} t_{j, k}(N) \\
& \quad \times H_{j, k}((1-\alpha-\beta) / 2) H_{j, k}((1+\alpha-\beta) / 2) \Xi_{+}(k-1 / 2 ; \alpha, \beta)
\end{aligned}
$$

with the function $\Xi_{+}$defined above by (43).

4.3. Back to the additive divisor problem. As before, let $\varrho \in \mathbb{C}$ with $0<\Re \varrho<1 / 2$. Using Proposition 1, we obtain an analytic continuation of the function $B^{\bullet}(-\varrho, \beta)$ (see (55)) from the domain

$$
R_{-\varrho}=\{\beta \in \mathbb{C}: \Re \beta<-3+\Re \varrho\}
$$

to a neighbourhood of the point $\beta=-\varrho$. It is evident from Sections 4.1 and 4.2 that the continuation is of a spectral nature. We propose a different look at $B^{\bullet}(-\varrho, \beta)$. 
Proposition 2. Let $W_{0}$ be nice enough. Assume that

$$
w_{0}(1)=\int_{0}^{1} W_{0}(x) d x=0, \quad w_{0}(1-\varrho)=\int_{0}^{1} W_{0}(x) x^{-\varrho} d x=0 .
$$

Then we have the representation

$$
\begin{aligned}
B^{\bullet}(-\varrho, \beta)= & N^{-\varrho} \sigma_{\beta}(N) \frac{\cot (\pi \varrho / 2)}{\pi} \zeta(-\varrho) w_{0}(-\varrho) \\
& -\sigma_{\beta}(N) \frac{\cot (\pi \varrho / 2)}{\pi} \zeta(\varrho) w_{0}(0)+\sigma_{\beta}(N) \zeta(-\beta) W^{-}(1) \\
& +\sum_{n=1}^{\infty} \sigma_{-\varrho}(n) \sigma_{\beta}(n+N) W^{+}(n / N) \\
& +\sum_{n=N+1}^{\infty} \sigma_{-\varrho}(n) \sigma_{\beta}(n-N) W^{-}(n / N) \\
& +\sum_{n=1}^{N-1} \sigma_{-\varrho}(n) \sigma_{\beta}(N-n) W^{-}(n / N),
\end{aligned}
$$

where for $x>0$,

$$
\begin{aligned}
W^{+}(x)= & -\frac{2 \cos (\pi \varrho / 2)}{\pi^{2}} \frac{1}{2 \pi i} \int_{(1 / 2)} w_{0}(s) \Gamma(s) \Gamma(1-s) \\
& \times \Gamma(s+\varrho) \Gamma(1-s-\varrho) \cos (\pi(s+\varrho / 2)) x^{-s} d s, \\
W^{-}(x)= & W_{0}(x)-\frac{2 \cos ^{2}(\pi \varrho / 2)}{\pi^{2}} \frac{1}{2 \pi i} \int_{(1 / 2)} w_{0}(s) \Gamma(s) \Gamma(1-s) \\
& \times \Gamma(s+\varrho) \Gamma(1-s-\varrho) x^{-s} d s .
\end{aligned}
$$

The representation (59) gives a meromorphic continuation of $B^{\bullet}(-\varrho, \beta)$ from $R_{-\varrho}$ to the domain $\Re \beta<0$, in particular to a neighbourhood of $-\varrho$.

From Propositions 1 and 2 we get immediately

Proposition 3. Let $W_{0}$ be nice enough and even (see (51)). Assume (58). Then

$$
\begin{aligned}
B_{\mathrm{h}}(-\varrho,-\varrho)= & B^{\bullet}(-\varrho,-\varrho) \\
= & N^{-\varrho} \sigma_{-\varrho}(N) \frac{\cot (\pi \varrho / 2)}{\pi} \zeta(-\varrho) w_{0}(-\varrho) \\
& +\sigma_{-\varrho}(N) \zeta(\varrho)\left(-\frac{\cot (\pi \varrho / 2)}{\pi} w_{0}(0)+W^{-}(1)\right)
\end{aligned}
$$




$$
\begin{aligned}
& +\sum_{n=1}^{\infty} \sigma_{-\varrho}(n) \sigma_{-\varrho}(n+N)\left(W^{+}(n / N)+W^{-}(1+n / N)\right) \\
& +\sum_{n=1}^{N-1} \sigma_{-\varrho}(n) \sigma_{-\varrho}(N-n) W^{-}(n / N),
\end{aligned}
$$

where $B_{\mathrm{h}}(-\varrho,-\varrho)$ is given by $(57)$ with $\alpha=\beta=-\varrho$, and for $W^{ \pm}$, see $(60)$ and (61) above.

Proof of Proposition 2. From (32), (33) and (55), for $\beta \in R_{-\varrho}$ we get (63)

$$
\begin{aligned}
B^{\bullet}(-\varrho, & \beta) \\
= & -\zeta(1-\beta) \sum_{l=1}^{\infty} l^{\beta-1} \sum_{1 \leq h \leq l,(h, l)=1} e(-N h / l) \frac{1}{2 \pi i} \int_{(-1 / 2)} N^{s} w_{0}(s) \\
& \times 2(2 \pi)^{2 s-2+\varrho} l^{-\varrho-2 s+1} \Gamma(1-s) \Gamma(1-\varrho-s) \\
& \times\left\{\cos (\pi \varrho / 2) D\left(1-s, \varrho ; e\left(h^{*} / l\right)\right)\right. \\
& \left.+\cos (\pi(s+\varrho / 2)) D\left(1-s, \varrho ; e\left(-h^{*} / l\right)\right)\right\} d s .
\end{aligned}
$$

Translate the integration line above to $\Re s=3 / 2$. Because of (58), there are only two poles of the integrand in the strip $-1 / 2 \leq \Re s \leq 3 / 2$, at $s=-\varrho$ and $s=0$. They come from the $D$-functions (see Lemma 1 ). Denote the contributions of $s=-\varrho$ and $s=0$ to $B^{\bullet}(-\varrho, \beta)$ by $G_{-\varrho}(\beta)$ and $G_{0}(\beta)$, respectively. From Lemma 1, (22), (23) and the functional equation of the Riemann zeta function it follows that

$$
\begin{aligned}
G_{-\varrho}(\beta)= & -N^{-\varrho} 2^{-\varrho} \pi^{-2-\varrho} \Gamma(1+\varrho) \cos (\pi \varrho / 2) \zeta(1+\varrho) w_{0}(-\varrho) \\
& \times \zeta(1-\beta) \sum_{l=1}^{\infty} l^{\beta-1} \sum_{1 \leq h \leq l,(h, l)=1} e(-N h / l) \\
= & N^{-\varrho} \sigma_{\beta}(N) \frac{\cot (\pi \varrho / 2)}{\pi} \zeta(-\varrho) w_{0}(-\varrho)
\end{aligned}
$$

and similarly

$$
G_{0}(\beta)=-\sigma_{\beta}(N) \frac{\cot (\pi \varrho / 2)}{\pi} \zeta(\varrho) w_{0}(0) .
$$

The remainder

$$
P(\beta)=B^{\bullet}(-\varrho, \beta)-G_{-\varrho}(\beta)-G_{0}(\beta)
$$

is given by the right hand side of (63) but with the new integration line $\Re s=3 / 2$.

Applying the functional equation (28) to $D\left(1-s, \varrho ; e\left( \pm h^{*} / l\right)\right)$ and the definition (27) of the $D$-functions, and changing the order of summation and 
integration, we get

$$
P(\beta)=P^{+}(\beta)+P^{-}(\beta)
$$

with

$$
P^{ \pm}(\beta)=\sum_{n=1}^{\infty} \sigma_{-\varrho}(n) \sigma_{\beta}(n \pm N) W^{ \pm}(n / N) .
$$

Here we put

$$
\sigma_{\beta}(0)=\zeta(-\beta)
$$

which is in accordance with the observation that for $\Re \beta<-1$,

$$
\zeta(1-\beta) \sum_{l=1}^{\infty} l^{\beta-1} \sum_{1 \leq h \leq l,(h, l)=1} 1=\zeta(-\beta)
$$

(cf. (22), (23)), and for $x>0$,

$$
\begin{aligned}
W^{+}(x)= & -\frac{2 \cos (\pi \varrho / 2)}{\pi^{2}} \frac{1}{2 \pi i} \int_{(3 / 2)} w_{0}(s) \Gamma(s) \Gamma(1-s) \\
& \times \Gamma(s+\varrho) \Gamma(1-s-\varrho) \cos (\pi(s+\varrho / 2)) x^{-s} d s,
\end{aligned}
$$

(71) $\quad W^{-}(x)$

$$
\begin{aligned}
= & \left(-\frac{1}{\pi^{2}}\right) \frac{1}{2 \pi i} \int_{(3 / 2)} w_{0}(s) \Gamma(s) \Gamma(1-s) \\
& \times \Gamma(s+\varrho) \Gamma(1-s-\varrho)\left(\cos ^{2}(\pi \varrho / 2)+\cos ^{2}(\pi(s+\varrho / 2))\right) x^{-s} d s \\
= & W_{0}(x)-\frac{2 \cos ^{2}(\pi \varrho / 2)}{\pi^{2}} \frac{1}{2 \pi i} \int_{(3 / 2)} w_{0}(s) \Gamma(s) \Gamma(1-s) \\
& \times \Gamma(s+\varrho) \Gamma(1-s-\varrho) x^{-s} d s .
\end{aligned}
$$

By translating the integration lines in (70) and (71) to $\Re s=1 / 2$ and using the assumption (58), we obtain (60) and (61). Put together (63)-(69) in order to get (59).

4.4. A special weight function. For $p \in \mathbb{C}$ let

$$
W_{p}(x)= \begin{cases}(x(1-x))^{p-1} & \text { if } 0<x<1, \\ 0 & \text { if } x \geq 1 .\end{cases}
$$

We remark that the same definition is given in Section 1.

An individual $W_{p}$-function cannot satisfy the assumption (58) of Propositions 2 and 3 , but a linear combination of $W_{p}$ 's can. For a later purpose (see Section 4.5) it is sufficient to consider linear combinations of just three 
$W_{p}$ 's. So, set

$$
W_{0}(x)=\sum_{i=1}^{3} c_{i} W_{p_{i}}(x) \quad(x>0)
$$

with $c_{1}, c_{2}, c_{3}, p_{1}, p_{2}, p_{3} \in \mathbb{C}$.

Assume that $\Re p_{i}(i=1,2,3)$ are sufficiently large. Then the above function $W_{0}$ is nice enough and even (see the beginning of Section 4.2 and (51)).

The Mellin transform of $W_{p}$ is

$$
w_{p}(s)=\int_{0}^{1} W_{p}(x) x^{s-1} d x=\frac{\Gamma(p) \Gamma(p+s-1)}{\Gamma(2 p+s-1)} \quad(\Re s>1-\Re p) .
$$

So, by (24) and (72)-(74),

$$
\begin{aligned}
w_{0}(s) & =\sum_{i=1}^{3} c_{i} w_{p_{i}}(s) \\
& =\sum_{i=1}^{3} c_{i} \frac{\Gamma\left(p_{i}\right) \Gamma\left(p_{i}+s-1\right)}{\Gamma\left(2 p_{i}+s-1\right)} \quad\left(\Re s>1-\Re p_{i} ; i=1,2,3\right) .
\end{aligned}
$$

The condition (58) of Propositions 2 and 3 is equivalent to the following linear system of two equations with respect to $c_{1}, c_{2}$ and $c_{3}$ :

$$
\begin{aligned}
\sum_{i=1}^{3} c_{i} \frac{\Gamma\left(p_{i}\right) \Gamma\left(p_{i}\right)}{\Gamma\left(2 p_{i}\right)} & =0 \\
\sum_{i=1}^{3} c_{i} \frac{\Gamma\left(p_{i}\right) \Gamma\left(p_{i}-\varrho\right)}{\Gamma\left(2 p_{i}-\varrho\right)} & =0 .
\end{aligned}
$$

Put

$$
\begin{aligned}
c_{1} & =\frac{\Gamma\left(p_{2}\right) \Gamma\left(p_{2}\right) \Gamma\left(p_{3}\right) \Gamma\left(p_{3}-\varrho\right)}{\Gamma\left(2 p_{2}\right) \Gamma\left(2 p_{3}-\varrho\right)}-\frac{\Gamma\left(p_{3}\right) \Gamma\left(p_{3}\right) \Gamma\left(p_{2}\right) \Gamma\left(p_{2}-\varrho\right)}{\Gamma\left(2 p_{3}\right) \Gamma\left(2 p_{2}-\varrho\right)}, \\
c_{2} & =\frac{\Gamma\left(p_{3}\right) \Gamma\left(p_{3}\right) \Gamma\left(p_{1}\right) \Gamma\left(p_{1}-\varrho\right)}{\Gamma\left(2 p_{3}\right) \Gamma\left(2 p_{1}-\varrho\right)}-\frac{\Gamma\left(p_{1}\right) \Gamma\left(p_{1}\right) \Gamma\left(p_{3}\right) \Gamma\left(p_{3}-\varrho\right)}{\Gamma\left(2 p_{1}\right) \Gamma\left(2 p_{3}-\varrho\right)}, \\
c_{3} & =\frac{\Gamma\left(p_{1}\right) \Gamma\left(p_{1}\right) \Gamma\left(p_{2}\right) \Gamma\left(p_{2}-\varrho\right)}{\Gamma\left(2 p_{1}\right) \Gamma\left(2 p_{2}-\varrho\right)}-\frac{\Gamma\left(p_{2}\right) \Gamma\left(p_{2}\right) \Gamma\left(p_{1}\right) \Gamma\left(p_{1}-\varrho\right)}{\Gamma\left(2 p_{2}\right) \Gamma\left(2 p_{1}-\varrho\right)} .
\end{aligned}
$$

This is of course a solution of (76).

Further on $W_{0}$ denotes the concrete function defined by (72), (73) and (77) under all the assumptions made.

Now we are going to determine an explicit form of the right and left hand sides of the formula (62) of Proposition 3 with our $W_{0}$. 
For $\Re p$ large enough and $\varrho \in \mathbb{C}$ with $0<\Re \varrho<1 / 2$ put, for $x>0$,

$$
\begin{aligned}
W_{p}^{+}(x)= & -\frac{2 \cos (\pi \varrho / 2)}{\pi^{2}} \frac{1}{2 \pi i} \int_{(1 / 2)} \frac{\Gamma(p) \Gamma(p+s-1)}{\Gamma(2 p+s-1)} \Gamma(s) \Gamma(1-s) \\
& \times \Gamma(s+\varrho) \Gamma(1-s-\varrho) \cos (\pi(s+\varrho / 2)) x^{-s} d s \\
W_{p}^{-}(x)= & W_{p}(x)+\widetilde{W}_{p}(x) \\
= & W_{p}(x)-\frac{2 \cos ^{2}(\pi \varrho / 2)}{\pi^{2}} \frac{1}{2 \pi i} \int_{(1 / 2)} \frac{\Gamma(p) \Gamma(p+s-1)}{\Gamma(2 p+s-1)} \\
& \times \Gamma(s) \Gamma(1-s) \Gamma(s+\varrho) \Gamma(1-s-\varrho) x^{-s} d s
\end{aligned}
$$

(cf. (60), (61), (72)-(75)).

\section{Lemma 3. We have}

(80) $\quad W_{p}^{+}(x)$

(81) $\quad \widetilde{W}_{p}(x)$

$$
\begin{aligned}
= & \frac{\cot (\pi \varrho / 2)}{\pi}\left(-\frac{\Gamma(p) \Gamma(p)}{\Gamma(2 p)} F(1, p ; 2 p ;-1 / x) x^{-1}\right. \\
& \left.+\frac{\Gamma(p) \Gamma(p-\varrho)}{\Gamma(2 p-\varrho)} F(1, p-\varrho ; 2 p-\varrho ;-1 / x) x^{\varrho-1}\right) \quad(x>0),
\end{aligned}
$$

$$
\begin{aligned}
= & \frac{\cot (\pi \varrho / 2)}{\pi}\left(\frac{\Gamma(p) \Gamma(p-1)}{\Gamma(2 p-1)} F(1, p ; 2-p ; 1-1 / x) x^{-1}\right. \\
& \left.-\frac{\Gamma(p-1) \Gamma(p-\varrho)}{\Gamma(2 p-1-\varrho)} F(1, p-\varrho ; 2-p ; 1-1 / x) x^{\varrho-1}\right) \quad(x>0),
\end{aligned}
$$

(82) $\quad \widetilde{W}_{p}(x)=\frac{\cot (\pi \varrho / 2)}{\pi}\left(\frac{\Gamma(p) \Gamma(p)}{\Gamma(2 p)} F(1, p ; 2 p ; 1 / x) x^{-1}\right.$

$$
\left.-\frac{\Gamma(p) \Gamma(p-\varrho)}{\Gamma(2 p-\varrho)} F(1, p-\varrho ; 2 p-\varrho ; 1 / x) x^{\varrho-1}\right) \quad(x>1),
$$

(83) $\quad W_{p}^{+}(x)+W_{p}^{-}(1+x)$

$$
\begin{aligned}
= & W_{p}^{+}(x)+\widetilde{W}_{p}(1+x) \\
= & \frac{\cot (\pi \varrho / 2)}{\pi} \frac{\Gamma(p) \Gamma(p-\varrho)}{\Gamma(2 p-\varrho)}\left(F(1, p-\varrho ; 2 p-\varrho ;-1 / x) x^{\varrho-1}\right. \\
& \left.-F(1, p ; 2 p-\varrho ;-1 / x)(1+x)^{\varrho} x^{-1}\right) \quad(x>0),
\end{aligned}
$$

(84) $W_{p}^{-}(1-x)$

$$
\begin{aligned}
= & (1+\cot (\pi \varrho / 2) \cot (\pi p)) W_{p}(x)-\frac{\cot (\pi \varrho / 2)}{\pi} \frac{\Gamma(p-\varrho) \Gamma(p-1)}{\Gamma(2 p-\varrho-1)} \\
& \times(1-x)^{\varrho} F(1,2+\varrho-2 p ; 2-p ; x)+N_{p}(x)
\end{aligned}
$$


with

$$
\begin{aligned}
N_{p}(x)= & \frac{2}{\pi} \cot (\pi \varrho / 2) \frac{\Gamma(p) \Gamma(p)}{\Gamma(2 p-1)} \\
& \times(1-2 x) F\left(1,3 / 2-p ; 3 / 2 ;(1-2 x)^{2}\right) \quad(0<x<1), \\
W_{p}^{-}(1)= & \widetilde{W}_{p}(1)
\end{aligned}
$$$$
=\frac{\cot (\pi \varrho / 2)}{\pi}\left(\frac{\Gamma(p) \Gamma(p-1)}{\Gamma(2 p-1)}-\frac{\Gamma(p-1) \Gamma(p-\varrho)}{\Gamma(2 p-1-\varrho)}\right) .
$$

Proof. From the identity

$$
\begin{aligned}
\cos (\pi(s+\varrho / 2)) \Gamma(1-s) \Gamma & (1-s-\varrho) \\
& =\frac{\pi}{2 \sin (\pi \varrho / 2)}\left(\frac{\Gamma(1-s)}{\Gamma(s+\varrho)}-\frac{\Gamma(1-s-\varrho)}{\Gamma(s)}\right)
\end{aligned}
$$

and (78) we get

$$
\begin{aligned}
W_{p}^{+}(x)= & -\frac{\cot (\pi \varrho / 2)}{\pi}\left(\frac{1}{2 \pi i} \int_{(1 / 2)} \frac{\Gamma(p) \Gamma(p+s-1) \Gamma(s) \Gamma(1-s)}{\Gamma(2 p+s-1)} x^{-s} d s\right. \\
& \left.-\frac{1}{2 \pi i} \int_{(1 / 2)} \frac{\Gamma(p) \Gamma(p+s-1) \Gamma(s+\varrho) \Gamma(1-s-\varrho)}{\Gamma(2 p+s-1)} x^{-s} d s\right) .
\end{aligned}
$$

This and (10) give (80) after some calculation.

By (79), for $x>0$,

$$
\begin{aligned}
\widetilde{W}_{p}(x)= & -\frac{2 \cos ^{2}(\pi \varrho / 2)}{\pi^{2}} \frac{1}{2 \pi i} \int_{(1 / 2)} \frac{\Gamma(p) \Gamma(p+s-1)}{\Gamma(2 p+s-1)} \Gamma(s) \Gamma(1-s) \\
& \times \Gamma(s+\varrho) \Gamma(1-s-\varrho) x^{-s} d s .
\end{aligned}
$$

Consider the above integral as the value of the convolution of $W_{p}$ and an appropriate hypergeometric function at $x$ (cf. (74) and (11)). Use further (13) and (16) to obtain successively, for $x>1$,

$$
\begin{aligned}
\widetilde{W}_{p}(x) & =-\frac{2 \cos ^{2}(\pi \varrho / 2)}{\pi^{2}} \Gamma(1+\varrho) \Gamma(1-\varrho) \int_{0}^{1} W_{p}(t) F(1,1-\varrho ; 2 ; 1-x / t) \frac{d t}{t} \\
& =-\frac{\cot (\pi \varrho / 2)}{\pi} \frac{\varrho}{x} \int_{0}^{1} W_{p}(t) F(1,1+\varrho ; 2 ; 1-t / x) d t \\
& =\frac{\cot (\pi \varrho / 2)}{\pi} \frac{1}{x} \int_{0}^{1} W_{p}(t) \frac{1-(t / x)^{-\varrho}}{1-t / x} d t .
\end{aligned}
$$

This, (72) and (9) imply (82).

Apply (12) to both terms of (82). The result is (81) valid for $x>1$. By analytic continuation, (81) holds for $x>0$. 
By (13), for $x>0$,

$$
F(1, p ; 2 p ; 1 /(x+1))(x+1)^{-1}=F(1, p ; 2 p ;-1 / x) x^{-1}
$$

and

$F(1, p-\varrho ; 2 p-\varrho ; 1 /(x+1))(x+1)^{\varrho-1}=F(1, p ; 2 p-\varrho ;-1 / x)(1+x)^{\varrho} x^{-1}$.

So (83) is a consequence of (80) and (82).

By (13), for $0<x<1$,

$$
\begin{aligned}
& F(1, p ; 2-p ; 1-1 /(1-x))(1-x)^{-1}=F(1,2-2 p ; 2-p ; x) \\
& F(1, p-\varrho ; 2-p ; 1-1 /(1-x))(1-x)^{\varrho-1} \\
& =F(1,2+\varrho-2 p ; 2-p ; x)(1-x)^{\varrho} .
\end{aligned}
$$

Use (17) to get, for $0<x<1$,

$$
\begin{aligned}
& F(1,2-2 p ; 2-p ; x) \\
& =\frac{\Gamma(2-p) \Gamma(1 / 2)}{\Gamma(3 / 2-p)} F\left(1 / 2,1-p ; 1 / 2 ;(1-2 x)^{2}\right) \\
& \quad+\frac{\Gamma(2-p) \Gamma(-1 / 2)}{\Gamma(1 / 2) \Gamma(1-p)}(1-2 x) F\left(1,3 / 2-p ; 3 / 2 ;(1-2 x)^{2}\right) .
\end{aligned}
$$

By (15),

(90) $\quad F\left(1 / 2,1-p ; 1 / 2 ;(1-2 x)^{2}\right)=2^{2 p-2}(x(1-x))^{p-1} \quad(0<x<1)$.

The formulas (84) and (85) now follow from (79), (81) and (87)-(90).

Take $x=1$ in (81) to get (86).

For $p, \varrho \in \mathbb{C}$ with $\Re p$ large enough, $0<\Re \varrho<1 / 2$ and $k$ even, $k \geq 6$ we introduce the notation

$$
\begin{aligned}
\Xi_{p}^{+}(k-1 / 2 ;-\varrho)= & \frac{1}{2 \pi i} \int_{(0)} \frac{\Gamma(k+\varrho-1+s)}{\Gamma(k+1-\varrho-s)} \Gamma(1-s) \\
& \times \Gamma(1-\varrho-s) w_{p}(s) \cos (\pi(s+\varrho / 2)) d s
\end{aligned}
$$

with $w_{p}(s)$ given by (74) (cf. (43), (57), (62), (72), (73), (75)).

LEMMA 4. Under the above assumptions

$$
\begin{aligned}
\Xi_{p}^{+}(k-1 / 2 ;-\varrho)= & -2^{2 \varrho-2 p+1} \frac{\Gamma(p) \Gamma(p-\varrho) \Gamma((k+\varrho) / 2)}{\Gamma(p+(k-\varrho) / 2) \Gamma((1+k-\varrho) / 2)} \\
& \times \Gamma(-p+(1+k+\varrho) / 2) \sin (\pi(p+(1-\varrho) / 2)) .
\end{aligned}
$$

Proof. Translate the integration line in (91) to $\Re s=+\infty$. The residue theorem, (8) and (74) give 


$$
\begin{aligned}
& \Xi_{p}^{+}(k-1 / 2 ;-\varrho)=\frac{\pi}{2 \sin (\pi \varrho / 2)} \\
& \quad \times\left(\frac{\Gamma(k+\varrho)}{\Gamma(k-\varrho) \Gamma(1+\varrho)} \int_{0}^{1} W_{p}(x) F(k+\varrho, 1-k+\varrho ; 1+\varrho ; x) d x\right. \\
& \left.\quad-\frac{1}{\Gamma(1-\varrho)} \int_{0}^{1} W_{p}(x) x^{-\varrho} F(k, 1-k ; 1-\varrho ; x) d x\right) .
\end{aligned}
$$

Apply (13) to the last hypergeometric function to get

$$
F(k, 1-k ; 1-\varrho ; x)=(1-x)^{-\varrho} F(k-\varrho, 1-k-\varrho ; 1-\varrho ; x) .
$$

From (18), (93) and (94) it follows that

$$
\begin{aligned}
& \Xi_{p}^{+}(k-1 / 2 ;-\varrho)=\frac{\pi}{2 \sin (\pi \varrho / 2)} \\
& \quad \times\left(\frac{\Gamma(k+\varrho) \Gamma(p) \Gamma(p)}{\Gamma(k-\varrho) \Gamma(1+\varrho) \Gamma(2 p)}{ }_{3} F_{2}(k+\varrho, 1-k+\varrho, p ; 1+\varrho, 2 p ; 1)\right. \\
& \quad-\frac{\Gamma(p-\varrho) \Gamma(p-\varrho)}{\Gamma(1-\varrho) \Gamma(2 p-2 \varrho)} \\
&\left.\quad \times{ }_{3} F_{2}(k-\varrho, 1-k-\varrho, p-\varrho ; 1-\varrho, 2 p-2 \varrho ; 1)\right) .
\end{aligned}
$$

Use (19) in order to evaluate the two ${ }_{3} F_{2}$ 's in (95). After some calculations we find that (92) holds.

For $\Re p$ large enough and $\varrho \in \mathbb{C}$ with $0<\Re \varrho<1 / 2$ denote by $B_{\mathrm{h}}$ the holomorphic part of the spectral decomposition for the binary additive divisor sum $B_{N}\left(-\varrho,-\varrho ; W_{p}\right)$ with $W_{p}$ given by (72). By (43), (57), (72), (74), (91), (92), the principal analytical properties of the $L$-functions $H_{j, k}$ (see [13, Lemma 3.6]), the estimate [13, (2.26)] and Stirling's asymptotic formula, we have

$$
B_{\mathrm{h}}=\mathfrak{H}_{N}(p, \varrho),
$$

where $\mathfrak{H}_{N}(p, \varrho)$ is defined by (1) in Section 2 .

Because of (73), the statement (96) implies

$$
B_{\mathrm{h}}(-\varrho,-\varrho)=\sum_{i=1}^{3} c_{i} \mathfrak{H}_{N}\left(p_{i}, \varrho\right) .
$$

We remark that here $c_{1}, c_{2}$ and $c_{3}$ can be any complex numbers, not just given by (77).

By (85), the function $N_{p}$ is odd with respect to $1 / 2$ :

$$
N_{p}(x)=-N_{p}(1-x) \quad(0<x<1) .
$$


Hence,

$$
\sum_{n=1}^{N-1} \sigma_{-\varrho}(n) \sigma_{-\varrho}(N-n) N_{p}(1-n / N)=0 .
$$

Use (8) and (83) to see that

$$
W_{p}^{+}(x)+W_{p}^{-}(1+x) \ll x^{-2+\Re \varrho} \quad(x \rightarrow+\infty) .
$$

From (60), (61), (72)-(75), (78), (79), (98), (99) and Lemma 3 it follows that the right hand side of (62) in Proposition 3 can be rewritten as

$$
B^{\bullet}(-\varrho,-\varrho)=\sum_{i=1}^{3} c_{i}\left(\mathfrak{G}_{N}\left(p_{i}, \varrho\right)+\mathfrak{A}_{N}\left(p_{i}, \varrho\right)+\mathfrak{D}_{N}\left(p_{i}, \varrho\right)\right)
$$

with $c_{1}, c_{2}$ and $c_{3}$ given by (77) and the notation introduced in Section 2 . Recall that (77) ensures that our $W_{0}$ satisfies the assumption (58) of Propositions 2 and 3 (cf. (75)-(77)).

Finally, from Proposition 3, (97) and (100) we obtain the following weak version of the Theorem.

Proposition 4. Let $N \in \mathbb{N}, \varrho, p_{1}, p_{2}, p_{3} \in \mathbb{C}$. Assume that

$$
0<\Re \varrho<1 / 2
$$

and

$$
\Re p_{i} \text { is sufficiently large } \quad(i=1,2,3) \text {. }
$$

Then

$$
\sum_{i=1}^{3} c_{i}\left(\mathfrak{H}_{N}\left(p_{i}, \varrho\right)-\mathfrak{G}_{N}\left(p_{i}, \varrho\right)-\mathfrak{A}_{N}\left(p_{i}, \varrho\right)-\mathfrak{D}_{N}\left(p_{i}, \varrho\right)\right)=0
$$

with $c_{1}, c_{2}$ and $c_{3}$ given by $(77)$.

4.5. End of proof of Theorem. We need a concrete version of the assumption (101) of Proposition 4.

Consider $\mathfrak{H}_{N}(p, \varrho)$ as a function of $p$. Convexity bounds for $H_{j, k}$ with respect to $k$ (cf. [13, Lemma 3.6]), the estimate

$$
\sum_{j=1}^{\vartheta(k)} \alpha_{j, k} \ll k
$$

(cf. $[12,(2.2 .10)]$ ) and Deligne's bound

$$
\left|t_{j, k}(N)\right| \leq d(N)=\sigma_{0}(N)
$$

can be applied to the series defining $\mathfrak{H}_{N}(p, \varrho)$, showing that this series is absolutely and uniformly convergent on compact subsets of the halfplane $\Re p>p_{0}$, where $p_{0}=3 / 2+\Re \varrho / 2$. Hence $\mathfrak{H}_{N}(p, \varrho)$ is regular there. 
Consider the series $\mathfrak{A}_{N}(p, \varrho)$ defined by (3) and (5). By using (8) and the ratio convergence test, we conclude that $\mathfrak{A}_{N}(p, \varrho) /(\Gamma(p) \Gamma(p-\varrho))$ is absolutely and uniformly convergent on compact subsets of the whole complex plane $\mathbb{C}$. Hence $\mathfrak{A}_{N}(p, \varrho) /(\Gamma(p) \Gamma(p-\varrho))$ is regular on $\mathbb{C}$.

Fix $x \in(0,1)$. Then $F(1,2+\varrho-2 p ; 2-p ; x) / \Gamma(2-p)$ is an entire function of $p$ (see [5, Subsection 2.1.6]). So the finite sum $\mathfrak{D}_{N}(p, \varrho)$, defined by (4) and (6), is meromorphic on $\mathbb{C}$. In order to see more, replace in (4) the $D_{p, q}$-function by the $W_{p}^{-}$-function given by (79). Observe that this does not change $\mathfrak{D}_{N}(p, \varrho)$. The function defined by the integral in (79) is regular in the halfplane $\Re p>1 / 2$. We conclude that $\mathfrak{D}_{N}(p, \varrho)$ is regular there.

It is clear from $(2)$ that $\mathfrak{G}_{N}(p, \varrho)$ is meromorphic on $\mathbb{C}$. It has only simple poles. In the halfplane $\Re p>1 / 2$ there are only two of them, at $p=1+\varrho$ and $p=1$. The respective residues are given in Corollary 1 .

We can therefore replace the assumption (101) of Proposition 4 by

$$
\Re p_{i}>p_{0}=3 / 2+\Re \varrho / 2 \quad(i=1,2,3) .
$$

Lemma 5. The equality (7) of the Theorem holds for $p=5 / 2+\varrho / 2$ and $p=7 / 2+\varrho / 2$.

Proof. Observe that with special $p=1 / 2+\varrho / 2+l, l$ being an integer, the series in (1) defining $\mathfrak{H}_{N}(p, \varrho)$ simplifies to a finite $\operatorname{sum} \sum_{k=6, k \text { even }}^{2 l}(\ldots)$ with a general term involving

$$
\sum_{j=1}^{\vartheta(k)} \alpha_{j, k} t_{j, k}(N) H_{j, k}(1 / 2) H_{j, k}(1 / 2+\varrho) .
$$

By the preceding discussion, it is also safe to assume that $l \geq 2$ (cf. (103)).

A trace formula of Bykovsky [3, Lemma 6] gives an arithmetical expression for (104); cf. also [10, Section 4.3], [11, Theorem 2.4], [2, Theorem 1], [7, Theorem 17]. (The results we are referring to are consequences of versions of Petersson's classical sum formulas; see, for example, [13, Lemma 2.3].) This arithmetical expression can be written in terms of the divisor function $\sigma_{s}(n)$ and the hypergeometric function. Its structure is quite similar to that of $\mathfrak{G}_{N}(p, \varrho)+\mathfrak{A}_{N}(p, \varrho)+\mathfrak{D}_{N}(p, \varrho)$, provided that $p=1 / 2+\varrho / 2+l, l$ being an integer. The lemma actually follows from the above result of Bykovsky by direct verification.

In Proposition 4 put $p_{1}=5 / 2+\varrho / 2, p_{2}=7 / 2+\varrho / 2$ and $p_{3}=p$ with $\Re p>3 / 2+\Re \varrho / 2$; cf. the discussion preceding Lemma 5 . This lemma and (102) give (7), because $c_{3} \neq 0$ in (77). The Theorem is proved. 


\section{References}

[1] D. Bump, S. Friedberg and J. Hoffstein, On some applications of automorphic forms to number theory, Bull. Amer. Math. Soc. 33 (1996), 157-175.

[2] V. A. Bykovsky, The trace formula for the scalar product of Hecke series and its applications, preprint, 1995 (in Russian).

[3] - Hecke series values of holomorphic cusp forms in the centre of the critical strip, in: Number Theory in Progress, Vol. 2, de Gruyter, 1999, 675-689.

[4] B. Conrey, L-functions and random matrices, in: Mathematics Unlimited-2001 and Beyond, Springer, 2001, 331-352.

[5] A. Erdélyi et al., Higher Transcendental Functions, Vol. I, McGraw-Hill, 1953.

[6] H. Iwaniec, Spectral Methods of Automorphic Forms, 2nd ed., Amer. Math. Soc., 2002 .

[7] H. Iwaniec and P. Sarnak, The non-vanishing of central values of automorphic Lfunctions and Landau-Siegel zeros, Israel J. Math. 120 (2000), 155-177.

[8] N. Katz and P. Sarnak, Zeros of zeta functions and symmetry, Bull. Amer. Math. Soc. 36 (1999), 1-26.

[9] N. V. Kuznetsov, Petersson hypothesis for parabolic forms of weight zero and Linnik hypothesis, Math. USSR-Sb. 39 (1981), 299-342.

[10] - Convolution of the Fourier coefficients of the Eisenstein-Maass series, Zap. Nauchn. Sem. LOMI 129 (1983), 43-84 (in Russian).

[11] -, On the mean value of Hecke series, preprint, 1994 (in Russian).

[12] Y. Motohashi, The binary additive divisor problem, Ann. Sci. École Norm. Sup. 27 (1994), 529-572.

[13] -, Spectral Theory of the Riemann Zeta-Function, Cambridge Univ. Press, 1997.

[14] M. Ram Murty and V. Kumar Murty, Non-vanishing of L-functions and Applications, Birkhäuser, 1997.

Faculty of Mathematics and Computer Science

A. Mickiewicz University

Umultowska 87

61-614 Poznań, Poland

E-mail: bszydlo@amu.edu.pl

Received on 18.3.2004

and in revised form on 6.11 .2006 